\title{
Carbon nanotube 3D current collectors for lightweight, high performance and low cost supercapacitor electrodes $\uparrow$
}

\author{
Ricardo Quintero, ${ }^{\text {ab }}$ Dong Young Kim, ${ }^{\mathrm{b}}$ Kei Hasegawa, ${ }^{\mathrm{b}}$ Yuki Yamada, ${ }^{\mathrm{a}}$ Atsuo Yamada ${ }^{\mathrm{a}}$ \\ and Suguru Noda*b
}

Self-supporting hybrid electrodes were fabricated through the systematic combination of activated carbon (AC), a low cost capacitive material, with sub-millimetre long few-wall carbon nanotubes (FWCNTs). After an easy three-step (mixing, dispersion and filtration) process, robust self-supporting films were obtained, comprising 90\% AC particles wrapped in a 3-dimensional FWCNT collector. The 10\% FWCNTs provide electrical conductivity and mechanical strength, and replace heavier metal collectors. The FWCNT matrix effectively improved the capacitance of the inexpensive, high surface area AC to $169 \mathrm{~F} \mathrm{~g}^{-1}$ at a slow scan rate of $5 \mathrm{mV} \mathrm{s}^{-1}$, and to $131 \mathrm{~F} \mathrm{~g}^{-1}$ at a fast scan rate of $100 \mathrm{mV} \mathrm{s}^{-1}$, in fairly thick $(\sim 200 \mu \mathrm{m})$ electrodes. Connection to a metallic collector at the film edge only, which significantly reduced the use of metal, retained much larger capacitance for the AC-FWCNT hybrid film $\left(107 \mathrm{~F} \mathrm{~g}^{-1}\right)$ than for the conventional AC electrode with binder and conductive filler $\left(3.9 \mathrm{~F} \mathrm{~g}^{-1}\right.$ ) at a practical voltage scan rate, $100 \mathrm{mV} \mathrm{s}^{-1}$. Transport measurements in three- and two-electrode cells show that the FWCNT matrix can greatly enhance the conductivity of the AC-based films.

Received 11th December 2013

Accepted 6th January 2014

DOI: 10.1039/c3ra47517d

www.rsc.org/advances lightweight electrode could bring manufacturing advantages and flexibility for thin-film applications.

Carbon nanotubes (CNTs) have been extensively studied. Owing to their high electrical conductivity, fair surface area and outstanding mechanical properties, they are attractive candidates for current collectors. Excellent performance in supercapacitor electrodes ${ }^{3}$ and Li-ion batteries ${ }^{4}$ has been demonstrated for single-wall CNTs (SWCNTs). SWCNTs can work as both conductive and capacitive materials, owing to their high electrical conductivity and fair surface area (up to $\sim 1300 \mathrm{~m}^{2} \mathrm{~g}^{-1}$ ); however their current price (up to $\sim 1000$ USD per $\mathrm{g}$ ) is a huge barrier to their practical use. Multi-wall CNTs (MWCNTs), however, are now mass-produced and available at low cost $(\sim 100$ USD per $\mathrm{kg}$ ), although they are less conductive and have insufficient surface area $\left(\sim 300 \mathrm{~m}^{2} \mathrm{~g}^{-1}\right)$. We have developed fluidized-bed chemical vapour deposition (CVD) and realised continuous and batch-production of sub-millimetre-long few-wall CNTs (FWCNTs) $^{5}$ and SWCNTs, ${ }^{6}$ respectively. By oxidizing these long FWCNTs (Ox.FWCNTs), self-supporting electrodes for high-power $\mathrm{Li}$ batteries were developed. ${ }^{7}$ High electronic conductivities were reported for these self-supporting electrodes $\left(\sim 100 \mathrm{~S} \mathrm{~cm}^{-1}\right)$ because of the long length $(\sim 400 \mu \mathrm{m})$ and high aspect ratio $(\sim 50000)$ of the FWCNTs. Additionally, the functionalisation of their surface with oxygen functional groups contributed to the capacitance (via Faradaic reactions) while the unoxidized inner walls of the CNTs ensured that the electrode retained high electrical conductivity. 


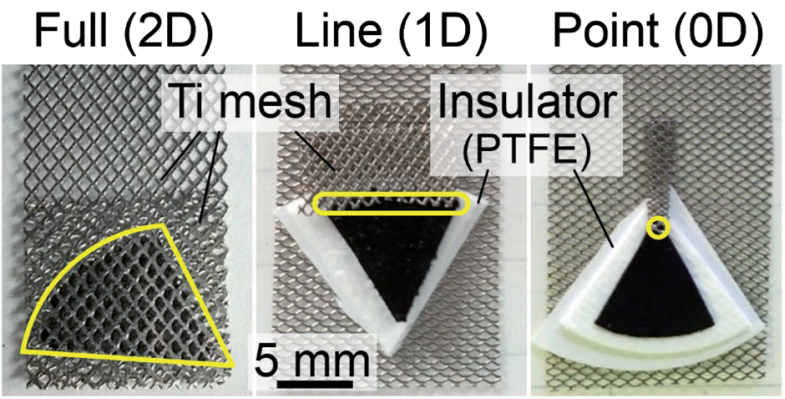

Fig. 1 Different configurations used to connect AC-FWCNT hybrid electrodes to $\mathrm{Ti}$ mesh. The highlighted areas indicate the contact between the Ti mesh and the carbon electrode.

Activated carbons (ACs) have high capacitances, because of their high specific surface area and large content of small pores. ${ }^{8,9}$ Their energy storage mechanism, which is based on the formation of an electrical double layer at their surface, is further enhanced by their surface chemistry, which includes oxygen functionalities that can provide a pseudocapacitance contribution. ${ }^{10}$ Their low cost and processability make them suitable for commercial supercapacitor devices when pasted (together with binders and conductive fillers) onto metal current collectors. But the resistance of these particles significantly affects device performance at fast charge rates. ${ }^{11}$ Polymer binders are normally needed to prepare AC-containing electrodes, but these binders then introduce high electrical resistance and reduce device performance. ${ }^{12}$

Electrochemical properties of electrodes are usually evaluated by placing the electrodes between metallic mesh current collectors or by coating a metallic plate/foil current collector with the active material, followed by immersion into an electrolyte. Because of this, the intrinsic resistance and performance of carbon electrodes without the collectors is not normally evaluated.

Reports featuring the combination of CNTs with capacitive materials such as transition metal oxides $\left(\mathrm{MnO}_{2}{ }^{13} \mathrm{RuO}_{2}\right.$ (ref. 14)), carbon nanohorns, ${ }^{15}$ graphite oxide, ${ }^{16,17}$ and $\mathrm{AC}^{18}$ indicate the feasibility of using CNTs to reduce the electrical series resistance (ESR) of composite electrodes; but, in all cases, the evaluation was performed using a metallic collector. Studies of CNTs used as

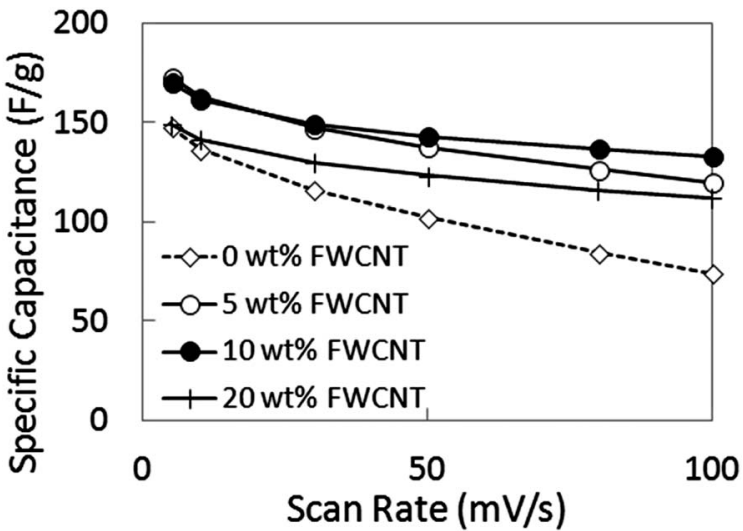

Fig. 3 Effects of different FWCNT loadings on the capacitance of ACFWCNT hybrid electrodes (three-electrode setup, full-contact Timesh configuration).

current collectors include the work of Niu et al. where they spread SWCNTs over a separator in a rolled configuration to act as both cathode and anode of a supercapacitor..$^{19} \mathrm{Hu}$ et al. used a CNT film as a current collector in thin and flexible paper for Li-ion batteries ${ }^{20}$ and Kaempgen et al. reported printable thin films of SWCNTs used as both electrodes and current collectors. ${ }^{21}$ Approaches combining CNTs and AC particles include a vacuum filtration method to prepare binder-free self-supporting electrodes from AC and SWCNTs in $1: 1$ ratio by Smithyman et al. ${ }^{22}$ Using a similar method, $\mathrm{Xu}$ et al. prepared binder-free AC/CNT paper with a $1-10 \%$ CNT loading. ${ }^{23}$ Chen et al. prepared ACcoated CNT paper through immersion of buckypaper in PAN precursor followed by carbonization, obtaining an optimum performance at a CNT loading of $75 \mathrm{wt} \%{ }^{24}$ The combination of capacitive AC particles with conductive CNTs is an effective way to obtain electrodes with high performance, but metallic-collectorfree $\mathrm{AC} / \mathrm{CNT}$ electrodes are yet to be reported.

In this work, inexpensive, capacitive AC particles were combined with conductive, flexible, long FWCNTs to obtain self-supporting conductive electrodes without any need for additional binder materials. The electrodes were characterized while connected in different ways to a Ti mesh to evaluate the performance of the CNT matrix as a current collector. Different
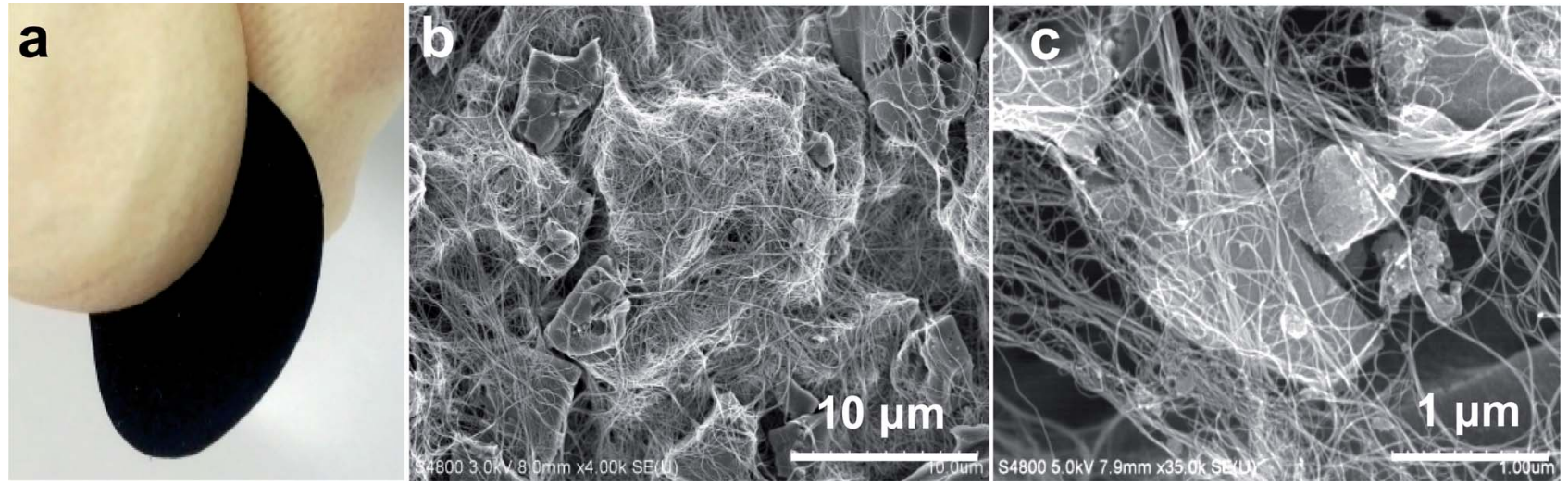

Fig. 2 Morphology of a typical AC-FWCNT electrode: (a) photograph, and (b, and c) SEM images. 
CNT contents were examined, and the capacitances of the electrodes were determined.

\section{Experimental}

\section{Electrode preparation}

Different amounts of FWCNTs (6-10 nm diameter, $400 \mu \mathrm{m}$ long, triple-wall on average $)^{5}$ were mixed with YP-80F activated carbon for supercapacitors (5-20 $\mu \mathrm{m}$ diameter, 1900-2200 $\mathrm{m}^{2}$ $\mathrm{g}^{-1}$ ) (Kuraray Chemical Co., Osaka, Japan) in AC : FWCNT weight ratios of $10: 0,9.5: 0.5,9: 1$ and $8: 2$. Both carbon materials were added to ethanol (EtOH) at $0.1 \mathrm{mg} \mathrm{mL}^{-1}$ concentrations and dispersed in a bath-type sonicator with a cooling unit to keep the temperature at $20{ }^{\circ} \mathrm{C}$. The electrodes were obtained by vacuum filtration over polytetrafluoroethylene (PTFE) membrane filters ( $5 \mu \mathrm{m}$ pore size) (see ESI, Fig. S1a $\dagger$ ). The final product was a mechanically-robust matrix of interwoven FWCNTs that held the AC particles. Residual solvent was removed by drying at $150{ }^{\circ} \mathrm{C}$ overnight, and typical final film densities were $\sim 0.25 \mathrm{~g} \mathrm{~cm}^{-3}$. Microstructure of the CNT-based films were characterized by scanning electron microscopy (SEM) S-4800 (Hitachi, Japan) and the specific surface areas were measured via the Brunauer-Emmett-Teller (BET) analysis of Nitrogen adsorption isotherms at $77 \mathrm{~K}$ (Quantachrome Instruments Autosorb 1C, FL, USA).

\section{Electrochemical characterization}

To evaluate the performance of the FWCNT 3D collector matrix, different configurations of a Ti mesh $(200 \mathrm{~L} \times 200 \mathrm{~S})$ connected to the carbon electrodes were prepared. The three Ti mesh configurations are shown in Fig. 1. The back side of the carbon electrodes were separated from the Ti mesh using a combination of PTFE and glass filter membranes so that the Ti mesh on the back side only serve as a structure to hold the insulated electrodes. The different ways of connection were named based on the area of the front of the electrodes covered by the Ti mesh: "full contact" or " $2 \mathrm{D}$ contact" when the Ti mesh covered the whole area of the electrode; "line contact" or " $1 \mathrm{D}$ contact" when the mesh was connected to an edge of the electrode; and "point contact" or " $0 \mathrm{D}$ contact" when the mesh was connected to the electrode at a single contact point. The goal of testing these configurations was to emulate the contacts inside a device, where the electrode and metallic collector meet at either an edge or a single point. Both two- and three-electrode cells were used to run cyclic voltammetry (CV), electrochemical impedance spectroscopy (EIS) and galvanostatic charge-discharge tests with a VMP3 potentiostat (Bio-Logic, Grenoble, France). The three-electrode cell consisted of an AC-FWCNT film as the working electrode, a Pt wire counter electrode and a $\mathrm{Ag} / \mathrm{AgCl}$ electrode (in saturated $\mathrm{NaCl}$ aqueous solution) reference electrode; the electrolyte was $1 \mathrm{M} \mathrm{H}_{2} \mathrm{SO}_{4}$ aqueous solution. For the
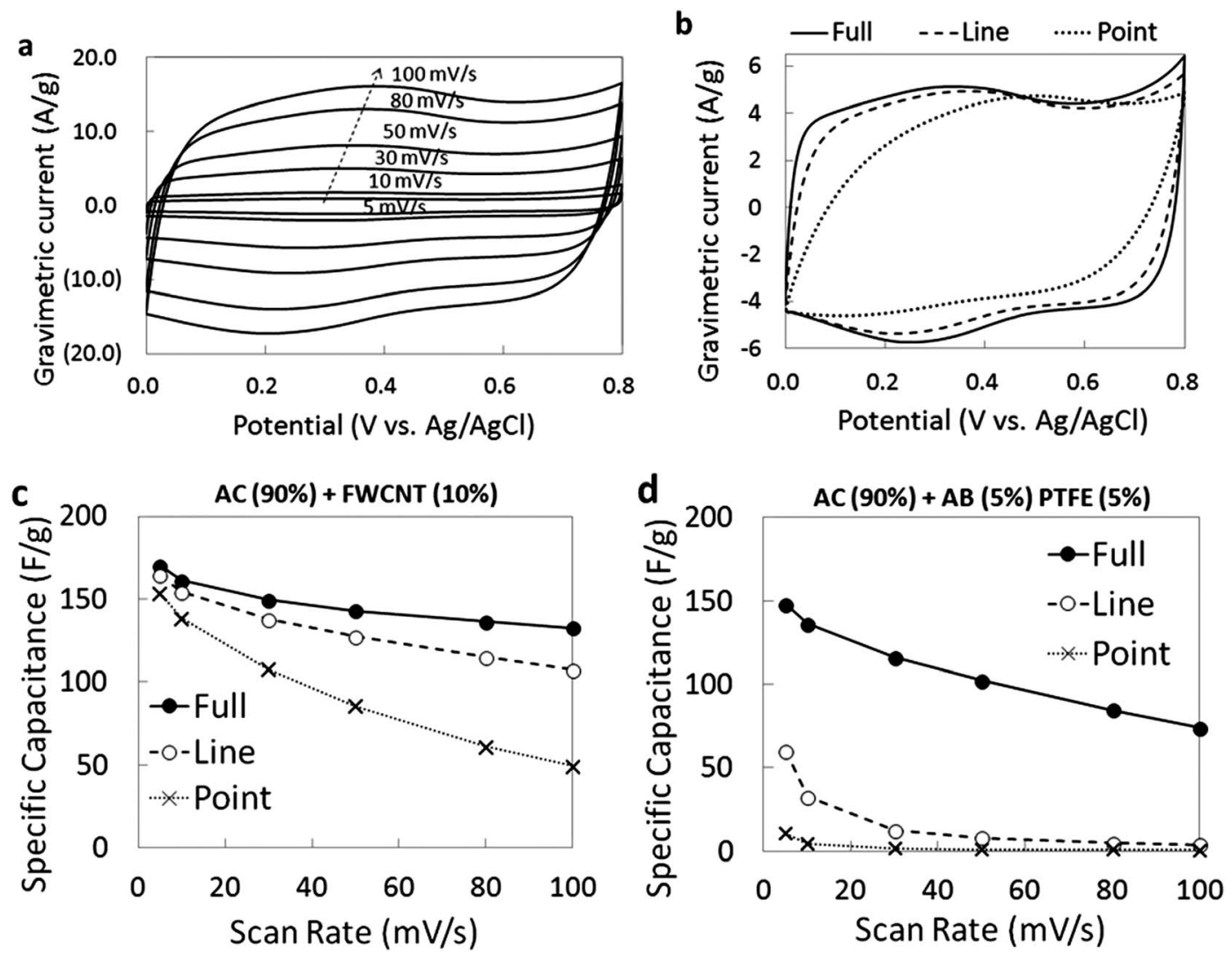

Fig. 4 (a-c) Electrochemical characterization of $\mathrm{AC}(90 \%)-\mathrm{FWCNT}(10 \%)$ hybrid electrodes using a three-electrode setup in $1 \mathrm{M} \mathrm{H}_{2} \mathrm{SO}_{4}$ : (a) $\mathrm{CV}$ plots at different scan rates for full-contact configuration; (b) CV plots at $30 \mathrm{mV} \mathrm{s}^{-1}$ and (c) scan-rate study $(0.0-0.8 \mathrm{~V}$ ), with different Ti-mesh configurations; (d) scan-rate study of electrodes containing AB filler and PTFE binder for the three Ti-mesh configurations. 
CVs, the electrodes were cycled 20 times at $30 \mathrm{mV} \mathrm{s}^{-1}$ prior to the measurement to exhaust any side reactions. ${ }^{25}$ The EIS tests were performed over a frequency range of $100 \mathrm{kHz}$ to $10 \mathrm{mHz}$ at a constant potential of $0.0 \mathrm{~V}$ vs. $\mathrm{Ag} / \mathrm{AgCl}$ (amplitude of the excitation signal $\pm 10 \mathrm{mV}$ ). The sheet conductivity of the selfsupporting films was evaluated through a 4-probe method.

\section{Results and discussion}

\section{Microstructure of the AC-FWCNT hybrid films}

Fig. 2 shows a photograph and SEM images of a typical ACFWCNT (9:1) electrode. The sponge-like matrix is the CNT 3D collector and the YP80F particles are encapsulated inside, yielding a flexible, self-supporting film. The long, flexible FWCNTs wrap around the AC particles in a continuous way, something that would not be possible with harder or shorter conductive materials. It must be noted that $\mathrm{EtOH}$ was selected as the solvent for the dispersion of both carbon species because of its ease of manipulation, good compatibility with AC particles and easy removal from the AC pores through post-annealing at low temperature. The simple three-step procedure-dispersion, filtration and drying-used to prepare the electrodes required no other reagents or additives such as binders, keeping the conductivity of the FWCNTs high. Since ultrasonication treatment of CNTs is known to readily reduce their length and quality with time, ${ }^{26}$ the conditions for this step were set to minimise shortening of the FWCNTs while, at the same time, achieving dispersion of AC particles.

The individual BET analyses of FWCNTs and YP-80F particles indicate specific surface areas of $428 \mathrm{~m}^{2} \mathrm{~g}^{-1}$ and $2034 \mathrm{~m}^{2}$ $\mathrm{g}^{-1}$ respectively (Fig. S2a $\dagger$ ), while the measured surface area of the AC-FWCNT hybrid electrode was $1783 \mathrm{~m}^{2} \mathrm{~g}^{-1}$, which is as expected from the individual contributions of both materials. It is commonly accepted that carbon materials with high surface areas can also display high capacitances since there is more area for charge accumulation and formation of the electric double layer. Still, other important factors such as electrical conductivity, pore distribution, electrolyte type and surface chemistry play major roles in the capacitance of a given material, and the relationship between specific capacitance and surface area is not linear. ${ }^{27}$ The Barrett-Joyner-Halenda (BJH) method for the calculation of the pore distribution in the ACFWCNT electrodes (Fig. S2b $\dagger$ ) indicates that most of the volume belongs to pores with diameters $<2 \mathrm{~nm}$, which can lead to good ion accessibility, depending on the electrolyte. ${ }^{28,29}$

\section{Basic performance of the AC-FWCNT hybrid films in three electrode setup}

The performance of the AC-based electrodes containing 0, 5, 10 or $20 \mathrm{wt} \%$ FWCNTs is shown in Fig. 3. The $0 \mathrm{wt} \%$ sample had a conventional supercapacitor-electrode composition and was prepared by mixing YP-80F particles (90 wt\%) with acetylene
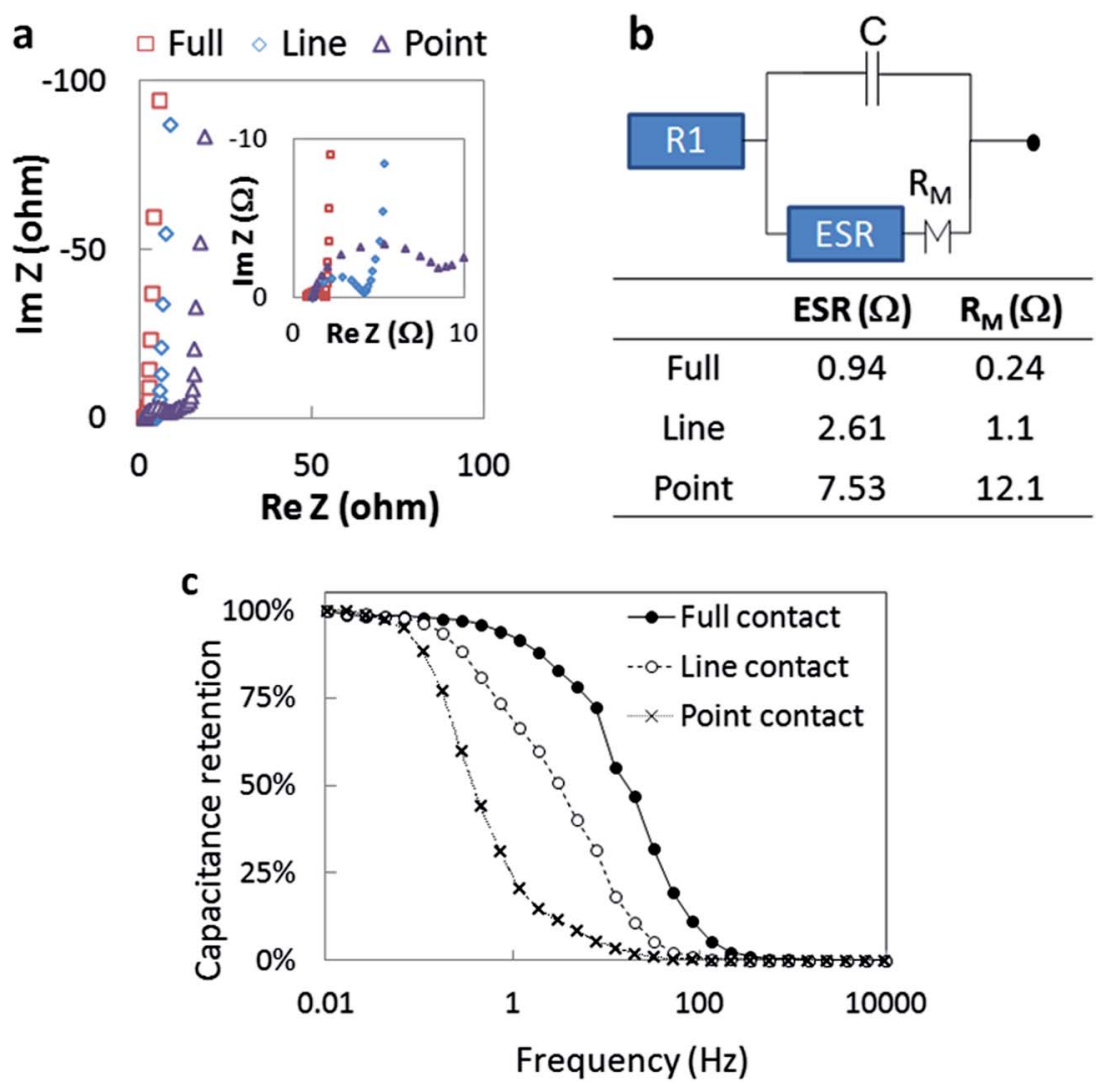

Fig. 5 (a) EIS results (100 kHz to $10 \mathrm{mHz}$ ) for the different Ti-mesh configurations in a three-electrode setup (Inset: high-frequency region); (b) equivalent electrical circuit with solution $\left(R_{1}\right)$, internal (ESR) and diffusion resistances $\left(R_{\mathrm{M}}\right)$; and (c) frequency-dependent capacitance retention. 
black $(\mathrm{AB})$ conductive filler (5 wt\%) and solid PTFE (5 wt\%) using a mortar and pestle. The results were obtained from $\mathrm{CV}$ tests in a three-electrode cell in the range $0.0-0.8 \mathrm{~V} v s . \mathrm{Ag} / \mathrm{AgCl}$, using the full-contact Ti-mesh configuration.

The specific capacitance was calculated from the CV plots using the expression:

$$
C=\frac{q}{v}=\frac{1}{v} \frac{\int_{E_{1}}^{E_{2}} i(E) \mathrm{d} E}{E_{2}-E_{1}}
$$

where $C$ is capacitance, $q$ is electric charge, $v$ is the scan rate, $E$ is voltage and $i$ is current. The mass of AC, FWCNT, and binder was used in the capacitance calculation.

Without FWCNTs, the capacitance of the AC decreases sharply at fast scan rates, to a minimum of $74 \mathrm{~F} \mathrm{~g}^{-1}$ at $100 \mathrm{mV}$ $\mathrm{s}^{-1}$. With addition of $5 \mathrm{wt} \%$ FWCNTs, there is a remarkable conductivity enhancement, leading to capacitance values of 172 and $119 \mathrm{~F} \mathrm{~g}^{-1}$ at 5 and $100 \mathrm{mV} \mathrm{s}^{-1}$, respectively. With $20 \mathrm{wt} \%$ addition, the capacitance decline with scan rate is less pronounced, although the maximum capacitance is reduced to $149 \mathrm{~F} \mathrm{~g}^{-1}$ at $5 \mathrm{mV} \mathrm{s}^{-1}$. A middle point is found at $10 \mathrm{wt} \%$ addition of FWCNTs, where the performance is highest across a wide scan-rate range $\left(170 \mathrm{~F} \mathrm{~g}^{-1}\right.$ at $5 \mathrm{mV} \mathrm{s}^{-1}$ and $132 \mathrm{~F} \mathrm{~g}^{-1}$ at $100 \mathrm{mV} \mathrm{s}^{-1}$ ) and the added amount of FWCNTs is still low enough that its contribution to the cost is small. As a result, a $10 \mathrm{wt} \%$ FWCNT load is adopted as the optimum for electrode preparation and the following tests in three- and two-electrode setups are carried out on $10 \mathrm{wt} \%$ FWCNT films.

The sheet conductivity of these electrodes versus the FWCNT content, and values from other studies of composites of MWCNT and SWCNT with AC, are plotted in Fig. S3. $\dagger$ The conductivities attained in the present work are very high considering the relatively low amounts of FWCNTs in the samples; this is because of the high aspect ratio of the FWCNTs used here. It should be noted that the value for $100 \mathrm{wt} \%$ FWCNT on the plot is obtained from FWCNT films cast from EtOH solution, and that a higher conductivity is achievable using a better dispersion method.

\section{AC-FWCNT hybrids with different contact configurations: evaluation by three-electrode setup}

The CV and scan-rate performance results for the three-electrode setup, with full-contact (2D), line-contact (1D) and pointcontact (0D) configurations (Fig. 1), are displayed in Fig. 4. The CVs exhibit broad oxidation and reduction peaks around $0.4 \mathrm{~V}$, which come from fast redox reactions. These reactions make a Faradaic contribution to the double-layer capacitance, and their effect is included in the overall capacitance.

The source of the redox peaks may be associated with the evolution and adsorption of activated oxygen groups on the carbon electrode surface and subsequent formation of quinoidal species. ${ }^{30}$

At low scan rate, the capacitance (in the voltage range evaluated) was the highest, at $169 \mathrm{~F} \mathrm{~g}^{-1}$ for the full-contact configuration. This higher capacitance at lower scan rates is typical for porous electrodes, where the pores deeper within the AC particles (which are 5-15 $\mu \mathrm{m}$ in diameter) and/or the film ( $200 \mu \mathrm{m}$ thick) contribute to capacitance at low scan rates only, depending on the mobility and diffusion of the ions within the electrolyte. ${ }^{31}$ With faster scan rates, fewer pores are able to contribute to capacitance, as the movement of ions is faster and less surface area is accessible. Also, the current has to be carried between the AC particles and current collectors through the FWCNTs, where the contact resistance governs the overall resistance. To enhance the overall conductivity of the film, the number of contact points between FWCNTs and AC particles in the parallel-circuit configuration should be increased by dispersing the AC particles and by wrapping the AC particles with FWCNTs. In addition, the number of contacts between the FWCNTs themselves, in a series-circuit configuration, can be decreased by enhancing the length of the FWCNTs. Because of this, longer FWCNTs can reduce the number of contact points in series, and compensate for the poor conductivity of the porous AC particles.

In the line-contact (1D) configuration, where the Ti mesh is connected to only one edge of the AC-FWCNT film, the rate performance shows similar values to those found for the fullcontact configuration at scan rates $\leq 30 \mathrm{mV} \mathrm{s}^{-1}$. At $100 \mathrm{mV} \mathrm{s}^{-1}$, the difference is still only about $20 \mathrm{~F} \mathrm{~g}^{-1}$, which shows the efficient movement of charge across the FWCNT 3D collector to its edge. The shape of the $\mathrm{CV}$ is also close to that of the fullcontact one, with the difference coming from the increased internal resistance of the electrode. The point-contact
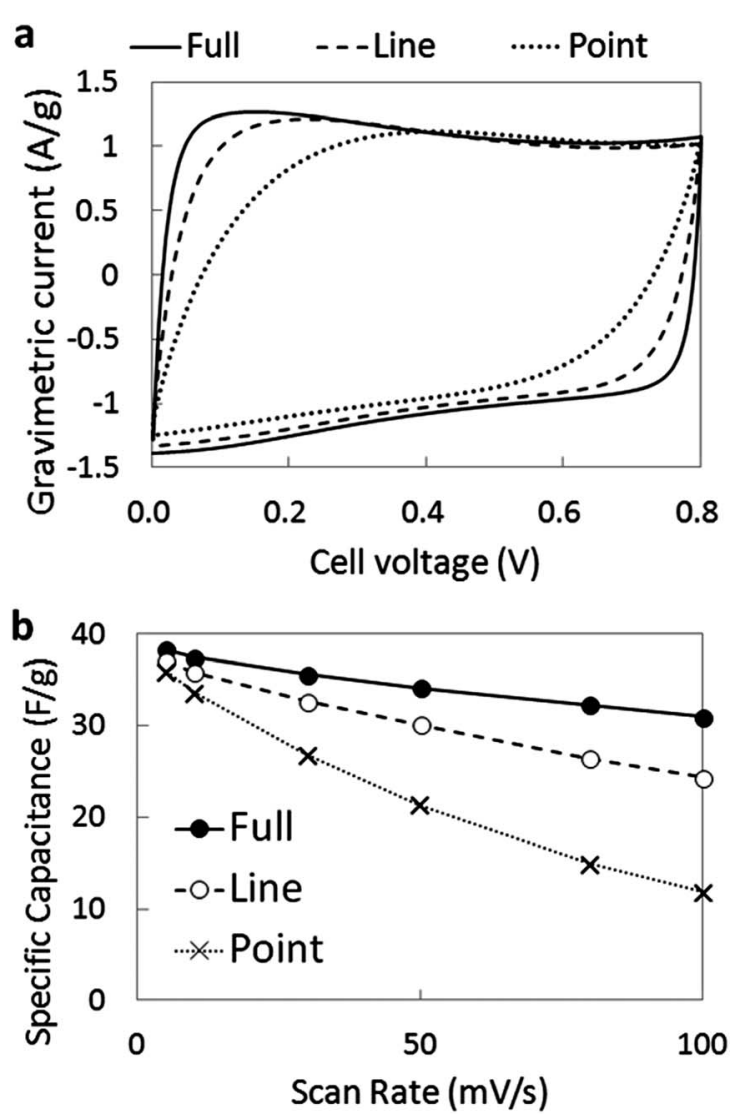

Fig. 6 (a) CV plots at $30 \mathrm{mV} \mathrm{s}^{-1}$; and (b) scan-rate study (0.0-0.8 V) for the different Ti-mesh configurations in a two-electrode setup. 
configuration displays the highest resistance, which causes a visible change in the shape of the CV curve. Even though the capacitance at $5 \mathrm{mV} \mathrm{s}^{-1}$ was surprisingly high $\left(153 \mathrm{~F} \mathrm{~g}^{-1}\right)$, meaning that the FWCNT matrix could transport most of the available charge through the electrode to one single point at a low rate, the sharp fall of the performance to $49 \mathrm{~F} \mathrm{~g}^{-1}$ at $100 \mathrm{mV}$ $\mathrm{s}^{-1}$ indicates the need to improve the conductivity of the FWCNT matrix in this configuration. The performance (evaluated with the three different Ti-mesh configurations) of an electrode made using conventional binder and conductive filler is displayed in Fig. 4d. For the full-contact configuration, the capacitance values are below those obtained for the samples containing FWCNTs in the same scan-rate range (for example, $74 \mathrm{~F} \mathrm{~g}^{-1}$ at $100 \mathrm{mV} \mathrm{s}^{-1}$ ). Worse still, the performance in lineand point-configurations is quite poor, with capacitances of $3.9 \mathrm{~F} \mathrm{~g}^{-1}$ and $0.5 \mathrm{~F} \mathrm{~g}^{-1}$ respectively at $100 \mathrm{mV} \mathrm{s}^{-1}$. We also examined AC-AB-PTFE electrodes with a higher filler content $(\mathrm{AB} 10 \mathrm{wt} \%$ ) and the use of a $\mathrm{Ti}$ foil as current collector (Fig. S4 $\dagger$ ). Compared with the AC (90 wt\%)-AB (5 wt\%)-PTFE (5 wt\%) electrode (Fig. 4d), the rate performance slightly improved owing to the increased content of the conducting fillers (i.e. $\mathrm{AB}$ ) while the capacitance at low rates slightly decreased due to the decreased content of the capacitive particles (i.e. AC). These results clearly show the need for a fullcontact metallic collector in the conventional sample, and highlight the role of the FWCNT matrix.
Nyquist diagrams from EIS analyses, shown in Fig. 5 for ACFWCNT and in Fig. S5 $\uparrow$ for AC-AB-PTFE, are important to determine the diffusion impedance of a porous electrode in electrochemical capacitors. They are normally made up of a semicircle (or part of a semicircle) at high frequencies, which corresponds to the electron-transfer-limited process, and a vertical line portion at low frequencies corresponding to the diffusion-limited process. Between these two regions, at moderate frequencies, there can be a transition zone, which is a straight line inclined at $45^{\circ}$, characteristic of a Warburg impedance, which is associated with the frequency-dependent diffusion of electrolyte into the pores. The solution resistance can be found by reading the real axis value at the high frequency intercept. The diameter of the semicircle corresponds to the equivalent series resistances or ESR of the electrode, which includes the contact between the active material and the current collector. In Fig. 5, the sizes of the semi-circles are different for every type of Ti-mesh configuration. For the full-contact configuration, the internal electrical resistance is the lowest $(0.94 \Omega)$, because the long-range electric conduction parallel to the electrode $(\sim 10 \mathrm{~mm})$ is largely performed by the Ti mesh, and only the short-range electronic conduction perpendicular to the electrodes $(\sim 200 \mu \mathrm{m})$ needs be done by the FWCNT matrix. The line-contact configuration shows a moderate resistance $(2.6 \Omega)$, since both the long-range electronic conduction parallel to and short-range electronic conduction perpendicular
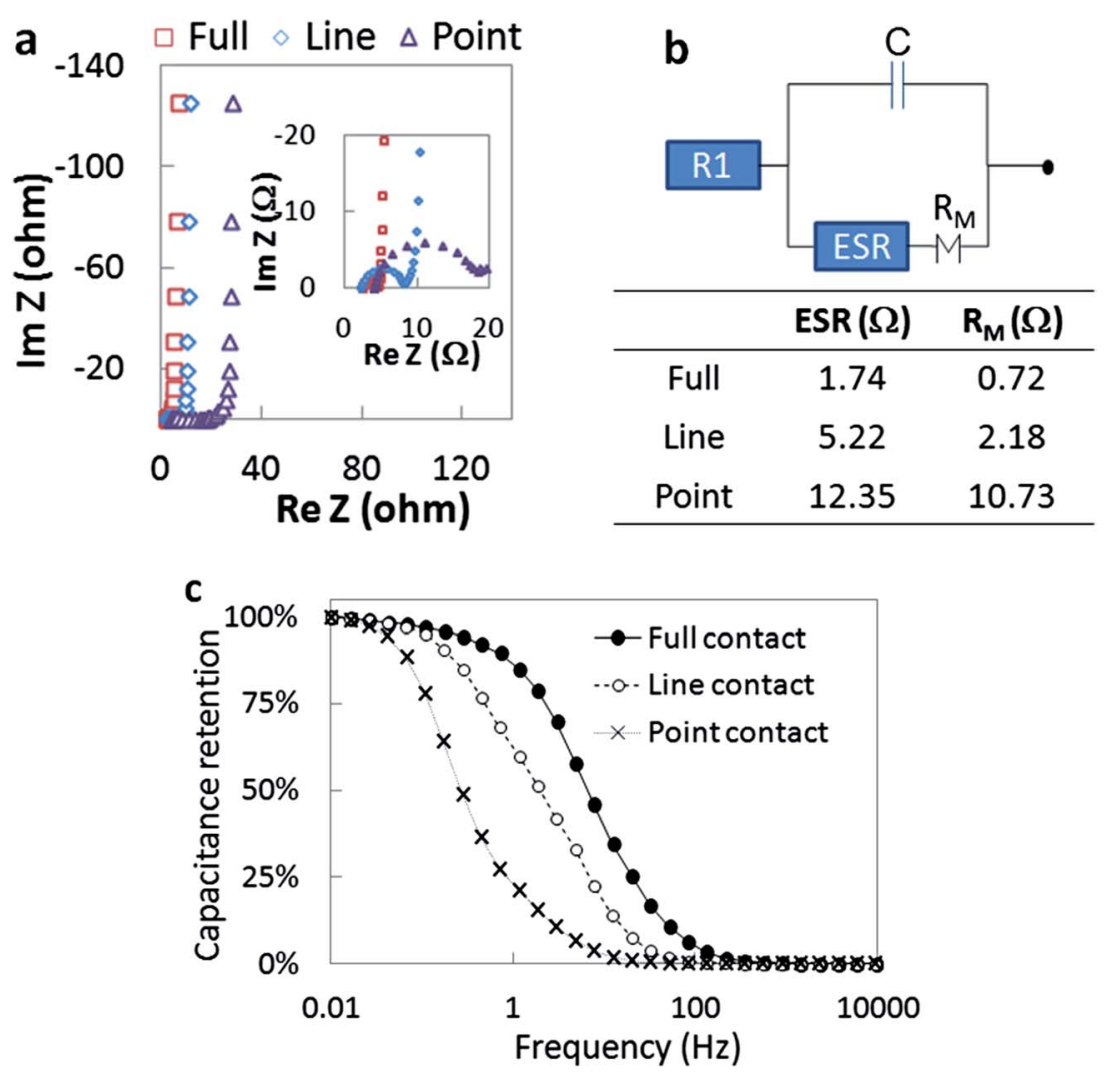

Fig. 7 Impedance characterisation in a two-electrode setup (a) EIS results (100 kHz to $10 \mathrm{mHz}$ ) for the different Ti-mesh configurations (Inset: high-frequency region); (b) equivalent electrical circuit with solution $\left(R_{1}\right)$, internal (ESR) and diffusion resistances $\left(R_{\mathrm{M}}\right)$; and $(\mathrm{c})$ frequencydependent capacitance retention. 
to the electrodes must be done by the FWCNT matrix (Fig. S1b $\dagger$ ). The point-contact configuration has the highest internal resistance $(7.5 \Omega)$. The difference between the ESR values for AC-FWCNT (0.94 $\Omega$, Fig. 5b) and AC-AB-PTFE (2.24 $\Omega$, Fig. S5c $\dagger$ ) is not particularly large $(1 / 2.4)$ in the full-contact configuration. These differences become much greater in the line- (2.6 vs. $83.7 \Omega$ ) and point- (7.5 vs. $236 \Omega$ ) configurations. The FWCNT 3D collector is effective in enhancing conduction, particularly the long-range electronic conduction parallel to the electrode (Fig. S1b $\dagger$ ).

Fig. 5c shows the capacitance retention as a function of frequency. The capacitance in this case is calculated from the imaginary part of the complex impedance. ${ }^{31}$

$$
C=-1 /(\omega \operatorname{Im}(Z))
$$

For all three Ti-mesh configurations, the capacitance retention is highest at low frequency. At high frequency, the electrolyte penetration depth is less than the actual pore length, and only a fraction of the surface area can contribute. It is possible to deduce the relaxation time constant $\left(\tau_{0}\right.$, which indicates the transition from capacitive behaviour to resistive behaviour) from the expression ${ }^{32}$

$$
\tau_{0}=1 / f_{0},
$$

where $f_{0}$ is the frequency at which the capacitance retention is $50 \%$.

The relaxation time constants for the electrodes with full-, line- and point-contact configurations are $0.059,0.33$, and $2.8 \mathrm{~s}$, respectively. In contrast, the corresponding values for the $\mathrm{AC}$ AB-PTFE electrode are $0.57,24.1$, and $43.5 \mathrm{~s}$, highlighting the role of FWCNTs in improving the electronic conduction through the electrode. In the full-contact configuration, electronic conduction and transport of ions vertically through the film and within individual AC particles are important. These transport mechanisms will be enhanced by reducing the film thickness and the particle size of AC, respectively. In the lineand point-contact configurations, in addition to those transport mechanisms, electronic conduction parallel to the film through the film becomes dominant. Such transport will be enhanced by improving dispersion and reducing damage to the FWCNTs during dispersion.

\section{AC-FWCNT hybrids with different contact configurations: evaluation by two-electrode setup}

Tests performed in a two-electrode cell measure in a more precise way the performance of electrodes for supercapacitor devices. The two-electrode setup should yield capacitance values $1 / 4$ of those found with the three-electrode setup. ${ }^{31}$

The CVs in Fig. 6 exhibit a semi-rectangular shape without defined redox peaks but with some broad signals present around 0.0 and $0.2 \mathrm{~V}$. For the full-contact configuration, the capacitance is highest at low scan rates, with a maximum of $40 \mathrm{~F}$ $\mathrm{g}^{-1}$ at $5 \mathrm{mV} \mathrm{s}^{-1}$, decreasing to $32.7 \mathrm{~F} \mathrm{~g}^{-1}$ at $100 \mathrm{mV} \mathrm{s}^{-1}$. The scan-rate study of the line-contact configuration shows similar behaviour to that found in the three-electrode setup, with good performance at low and moderate frequencies $\left(37 \mathrm{~F} \mathrm{~g}^{-1}\right.$ at $5 \mathrm{mV}$ $\mathrm{s}^{-1}$ and $30 \mathrm{~F} \mathrm{~g}^{-1}$ at $50 \mathrm{mV} \mathrm{s}^{-1}$ ). The point-contact configuration showed high capacitance in the slowest scan $\left(36 \mathrm{~F} \mathrm{~g}^{-1}\right.$ at $5 \mathrm{mV}$ $\mathrm{s}^{-1}$ ). Despite the high ESR of this configuration, a capacitance of $12 \mathrm{~F} \mathrm{~g}^{-1}$ at $100 \mathrm{mV} \mathrm{s}^{-1}$ was still achieved, which is impressive considering the low amount of FWCNT (10 wt\%) and the test conditions (point contact). The magnitudes of the contact resistances determined from the Nyquist diagrams in Fig. 7 are in agreement with the resistances measured in the three-electrode setup. Relaxation time constants of $0.17,0.5$ and $3.7 \mathrm{~s}$ were calculated for the full-, line- and point-configurations, respectively, using the frequency-dependent capacitance retention plots.

Stability and performance changes were tested by galvanostatic charge-discharge cycling between 0.0 and $0.8 \mathrm{~V}$ at a discharge current of $1 \mathrm{Ag}^{-1}$ (Fig. 8). The symmetric shape of the charge-discharge profiles is very close to that expected for an ideal EDLC, with small IR drops in the full- and line-configurations. In contrast, the point configuration displays a significant IR drop. After 1000 charge-discharge cycles, no weakening of the capacitance was observed, and the final capacitance values for the full-, contact- and point-configurations were 36 , 34.3 and $29.7 \mathrm{~F} \mathrm{~g}^{-1}$, respectively, at $1 \mathrm{~A} \mathrm{~g}^{-1}$ charge-discharge rate.

The capacitances obtained with the line- and point-contact configurations demonstrate that the FWCNT matrix is an
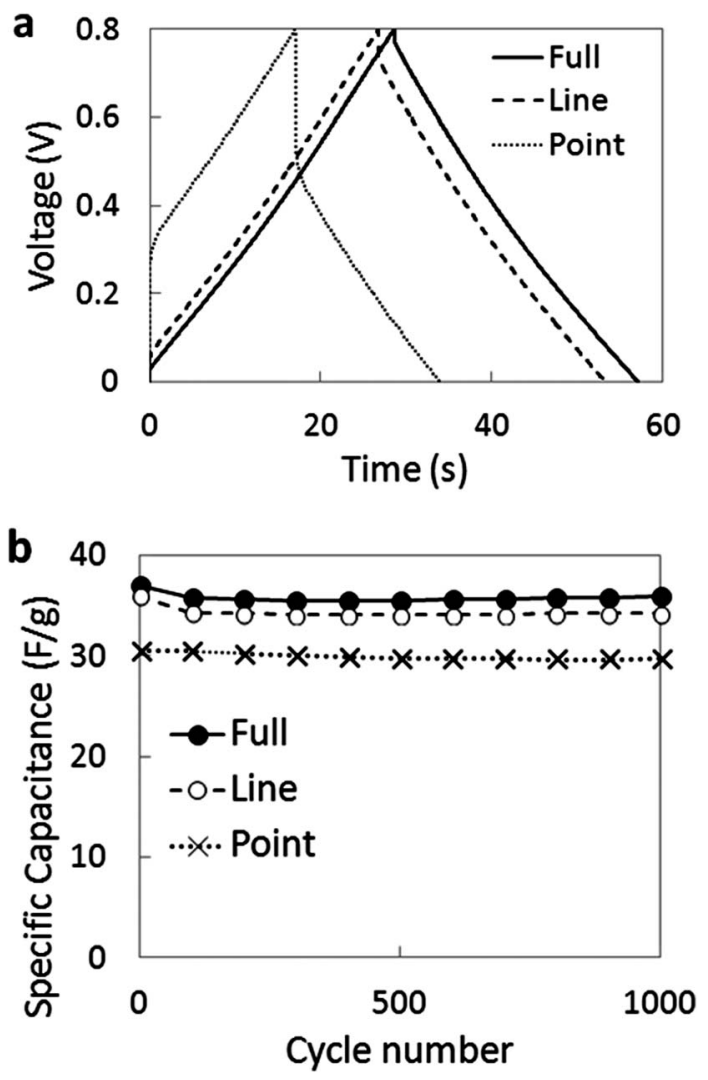

Fig. 8 Tests at $1 \mathrm{~A} \mathrm{~g}^{-1}$ on AC-FWCNT hybrid electrodes in $1 \mathrm{M} \mathrm{H}_{2} \mathrm{SO}_{4}$ with different Ti-mesh configurations: (a) galvanostatic chargedischarge plots; and (b) cycling stability tests. 
effective collector, and can partially or almost completely replace the metallic collector, depending on the desired application. Improvement of the electrical conductivity of the hybrid electrodes through better dispersion of the FWCNTs should further boost their performance.

\section{Conclusions}

We obtained self-supporting all-carbon hybrid electrodes by enclosing highly-capacitive activated carbon particles within an electrically conductive 3D collector made of FWCNTs. Electrical conductivity was enhanced after the AC particles were wrapped and bridged in the continuous matrix. The performance was evaluated in three different configurations: with a metallic collector on the full surface, on one edge, and on a single point of the hybrid films, and confirmed the feasibility of using FWCNTs as a high surface area 3D collector in a fairly thick film $\left(\begin{array}{ll}\sim 200 & \mu \mathrm{m}\end{array}\right)$. The sub-millimetre long FWCNTs provides mechanical stability and fewer contact and junction resistances, making it possible to produce self-supporting electrodes with no additional binder materials. The FWCNTs are able to hold nine times their own weight in AC capacitive particles. The fabrication method followed in this work can rapidly and easily produce lightweight electrodes with controlled thicknesses for varied applications, given its important advantage over other, more complicated, procedures.

This FWCNT conductive matrix should be ideal to enclose capacitive particles of any resistivity, such as transition metal oxides, to produce electrodes for high performance energystorage devices.

\section{Acknowledgements}

The authors thank Prof. Kuroda, Prof. Shimojima, and Mr Yamamoto for their support with the BET measurements and Kuraray Co., Ltd. for AC particles. This work was supported in part by Grant-in-Aid for Scientific Research (A) (no. 25249111) from JSPS, Japan, and by the ALCA Program from JST, Japan.

\section{Notes and references}

1 P. Simon and Y. Gogotsi, Nat. Mater., 2008, 7, 845.

2 S. W. Lee, B.-S. Kim, S. Chen, Y. Shao-Horn and P. T. Hammond, J. Am. Chem. Soc., 2008, 131, 671.

3 D. N. Futaba, K. Hata, T. Yamada, T. Hiraoka, Y. Hayamizu, Y. Kakudate, O. Tanaike, H. Hatori, M. Yumura and S. Iijima, Nat. Mater., 2006, 5, 987.

4 C. de las Casas and W. Li, J. Power Sources, 2012, 208, 74.

5 D. Y. Kim, H. Sugime, K. Hasegawa, T. Osawa and S. Noda, Carbon, 2011, 49, 1972.

6 D. Y. Kim, H. Sugime, K. Hasegawa, T. Osawa and S. Noda, Carbon, 2012, 50, 1538.

7 S. W. Lee, B. M. Gallant, Y. Lee, N. Yoshida, D. Y. Kim, Y. Yamada, S. Noda, A. Yamada and Y. Shao-Horn, Energy Environ. Sci., 2012, 5, 5437.
8 O. Barbieri, M. Hahn, A. Herzog and R. Kötz, Carbon, 2005, 43, 1303.

9 L. Wang, M. Toyoda and M. Inagaki, New Carbon Mater., 2008, 23, 111.

10 V. Ruiz, C. Blanco, E. Raymundo-Piñero, V. Khomenko, F. Beguin and R. Santamaría, Electrochim. Acta, 2007, 52, 4969.

11 R. Chandrasekaran, Y. Soneda, J. Yamashita, M. Kodama and H. Hatori, J. Solid State Electrochem., 2008, 12, 1349.

12 W. Lu, R. Hartman, L. Qu and L. Dai, J. Phys. Chem. Lett., 2011, 2, 655.

13 S. W. Lee, J. Kim, S. Chen, P. T. Hammond and Y. ShaoHorn, ACS Nano, 2010, 4, 3889.

14 Y.-T. Kim, K. Tadai and T. Mitani, J. Mater. Chem., 2005, 15, 4914.

15 A. Izadi-Najafabadi, T. Yamada, D. N. Futaba, M. Yudasaka, H. Takagi, H. Hatori, S. Iijima and K. Hata, ACS Nano, 2011, $5,811$.

16 S. H. Aboutalebi, A. T. Chidembo, M. Salari, K. Konstantinov, D. Wexler, H. K. Liu and S. X. Dou, Energy Environ. Sci., 2011, 4, 1855.

17 L. Qiu, X. Yang, X. Gou, W. Yang, Z.-F. Ma, G. G. Wallace and D. Li, Chem. - Eur. J., 2010, 16, 10653.

18 C. Lei and C. Lekakou, Surf. Coat. Technol., 2013, 232, 326.

19 Z. Niu, W. Zhou, J. Chen, G. Feng, H. Li, W. Ma, J. Li, H. Dong, Y. Ren, D. Zhao and S. Xie, Energy Environ. Sci., 2011, 4, 1440.

20 L. Hu, H. Wu, F. La Mantia, Y. Yang and Y. Cui, ACS Nano, 2010, 4, 5843.

21 M. Kaempgen, C. K. Chan, J. Ma, Y. Cui and G. Gruner, Nano Lett., 2009, 9, 1872.

22 J. Smithyman, A. Moench, R. Liang, J. Zheng, B. Wang and C. Zhang, Appl. Phys. A: Mater. Sci. Process., 2012, 107, 723.

23 G. Xu, C. Zheng, Q. Zhang, J. Huang, M. Zhao, J. Nie, X. Wang and F. Wei, Nano Res., 2011, 4, 870.

24 H. Chen, J. Di, Y. Jin, M. Chen, J. Tian and Q. Li, J. Power Sources, 2013, 237, 325.

25 M. D. Stoller and R. S. Ruoff, Energy Environ. Sci., 2010, 3, 1294.

26 J. Hilding, E. A. Grulke, Z. George Zhang and F. Lockwood, J. Dispersion Sci. Technol., 2003, 24, 1.

27 R. Signorelli, D. C. Ku, J. G. Kassakian and J. E. Schindall, Proc. IEEE, 2009, 97, 1837.

28 J. Kang, J. Wen, S. H. Jayaram, X. Wang and S.-K. Chen, J. Power Sources, 2013, 234, 208.

29 M. Seredych, M. Koscinski, M. Sliwinska-Bartkowiak and T. J. Bandosz, ACS Sustainable Chem. Eng., 2013, 1, 1024.

30 K.-W. Kim, M. Kuppuswamy and R. F. Savinell, J. Appl. Electrochem., 2000, 30, 543-549.

31 R. Kötz and M. Carlen, Electrochim. Acta, 2000, 45, 2483.

32 C. Portet, P. L. Taberna, P. Simon and E. Flahaut, J. Power Sources, 2005, 139, 371. 